\title{
SOSTENIBILIDAD DE Dalea lutea EN BOSQUE MIXTO Y PASTIZAL EN TENANCINGO, ESTADO DE MÉXICO ${ }^{1}$
}

\author{
Pedro Saldívar-Iglesias ${ }^{2}$,Antonio Laguna-Cerda², Claudio Esquivel-Álvarez ${ }^{2}$, Carlos González-Esquivel ${ }^{3}$
}

\section{RESUMEN}

Sostenibilidad de Dalea lutea en bosque mixto y pastizal en Tenancingo, Estado de México. El objetivo del presente trabajo fue analizar la dinámica poblacional, el comportamiento anual y evaluar la sostenibilidad ecológica de una población de D. lutea (Cav.) Willd. La presente investigación se llevó a cabo en la comunidad de Chalchihuapán, Tenancingo Estado de México (México) durante el periodo de otoño 2002 a otoño 2004. Se evaluó una población de Dalea lutea (Cav.) Willd. en bosque mixto y en pastizal inducido. Se marcaron para ello cuatro parcelas por zona y se clasificaron los individuos en tres tipos: plántulas, juveniles y adultas; los datos se trataron con análisis de varianza, prueba de medias y fórmulas de sostenibilidad. La población de $D$. lutea aumentó durante el verano-otoño y disminuyó durante el periodo invierno-primavera. Los muestreos presentaron diferencias estadísticas para plántulas y juveniles, no así en adultas, pero cuando estas clases se evaluaron dentro de las zonas no difirieron estadísticamente; el mayor número de individuos se reportó durante el verano, otoño e invierno de 2003. Respecto a sostenibilidad, el pastizal mostró al final del periodo un restablecimiento de 59,5 y $41 \%$ para plántulas y plantas adultas, respectivamente, y una disminución de $79 \%$ en juveniles. Mientras que en bosque mixto las plántulas y las juveniles decrecieron en 4,4 y 16,2\%, y las adultas se restablecieron en $25,1 \%$. La población de $D$. lutea aumentó o disminuyó conforme la época de muestreo, y el número de individuos estuvo afectado por la precipitación. Bajo las condiciones actuales de recolecta, la población de $D$. lutea se mantuvo estable en bosque mixto y se restableció mejor en la zona de pastizal.

Palabras clave: Cestería, dinámica poblacional, productos forestales no maderables.

\begin{abstract}
Sustainability of Dalea lutea at mixed forest and grassland in Tenancingo, Mexico State. The goals of this study were to assess the behavior, dynamics and sustainability of populations of Dalea lutea (Cav.) Willd. in mixed forest and grassland under natural conditions and of recollection. The investigation was carried out in a pine-oak forest near Tenancingo, Mexico State (Mexico), from Autumn 2002 to Autumn 2004. Four plots were marked in each zone and the individuals counted were classified in three types: seedlings, young and adults; the data were subject to analysis of variance, test of means and sustainability formulae. The population Dalea lutea increased in summer-autumn and decreased in winter-spring. Samplings showed statistical differences for seedlings and young, but not for adults, but when these classes were evaluated within zones they did not differ statistically; the largest number of individuals was reported during summer, autumn and winter of 2003. Regarding sustainability, there was a reestablishment within the pasture at the end of the period of 59.5 and $41 \%$ for seedlings and adults, respectively, and a reduction of $79 \%$ in young individuals; in the mixed forest, seedlings and the young plants decreased by 4.4 and $16.2 \%$, while adults recovered in $25.1 \%$. The population of Dalea lutea increased or decreased according to the time of sampling, and the number of individuals was determined by precipitation. Under the current conditions of collection, $D$. lutea population remains stable in the mixed forest and it is recovering better in the grassland.
\end{abstract}

Key words: Basketry, non-timber forest products, population dynamics.

Recibido: 13 de julio, 2011. Aceptado: 12 de marzo, 2012. Investigación doctoral del primer autor.

2 Facultad de Ciencias Agrícolas, Universidad Autónoma del Estado de México. Campus Universitario El Cerrillo, Km. 15 Carretera TolucaIxtlahuaca, entronque al Cerrillo, Apdo. Postal 435. Toluca Estado de México.psaldivari@yahoo.com.mx, alagunac@uaemex.mx

3 Centro de Investigaciones en Ecosistemas, Universidad Nacional Autónoma de México. Antigua Carretera a Pátzcuaro 8701 Ex-hacienda San José de la Huerta, Morelia, Michoacán CP 58190 México. cgesquivel@oikos.unam.mx 


\section{INTRODUCCIÓN}

La sostenibilidad es una corriente científica que plantea la necesidad de tener una visión integral y armónica del uso y conservación de los recursos naturales, por lo que busca compatibilizar la calidad de vida de los productores con la protección al ambiente, incorporando en ello elementos éticos en la conservación. El manejo sustentable de recursos naturales se define como el conjunto de acciones que tienden a satisfacer en forma indefinida la creciente demanda de satisfactores a costos económicos, ambientales y socialmente aceptables, asegurando su permanencia para la presente y futuras generaciones (Carabias et al. 1995, Casas 1996, Kammerbauer 2001, Matteucci y Colma 1997). En relación a la sostenibilidad existen visiones complementarias: la ecológica pugna por el uso adecuado de los recursos naturales y la conservación de la biodiversidad, buscando que las actividades humanas no sobrepasen la capacidad de regeneración de los ecosistemas; la económica se fundamenta en proveer ingresos estables a las comunidades rurales, cuidando de no exceder la capacidad productiva de las poblaciones (Hall y Bawa 1993). Por su parte, Pimentel y Castañeta (2007) mencionan que la sostenibilidad es un proceso que permite el acceso al bienestar y por lo tanto tiene carácter social, económico y biológico.

La recolecta y uso de productos del bosque, campos de cultivo y jardines domésticos es una estrategia en muchas culturas para obtener alimentos o recursos económicos adicionales (Sundriyal y Sundriyal 2001). La recolección de productos forestales no maderables (PFNM) representa una fuente de ingresos para las personas que viven en o cerca del bosque (Gómez y Angón 2004, Anónimo 2001). Se considera como recolección, la extracción de frutas, semillas, látex, fibras, ceras, resinas, gomas, raíces o cualquier producto de interés económico que pueda ser comercializado en mercados locales, regionales o nacionales (SARH 1994, Velásquez 1998). En los últimos años se ha observado un creciente interés y una demanda mayor en relación a los PFNM y se ha tomado conciencia de su potencial comercial prácticamente desaprovechado, pero un tema constante, ha sido la amenaza que plantea la sobreexplotación, ya que la viabilidad comercial de un producto dependerá de su disponibilidad, pero para asegurar una oferta regular, es necesario contar con el conocimiento cabal de la capacidad de regeneración de tal producto, de modo que se pueda mantener un equilibrio entre esta y la escala de producción (Pimentel y Castañeta 2007). En el caso de muchos PFNM no existen datos estadísticos suficientes para determinar si un recurso está siendo aprovechado en forma adecuada o si su explotación pone en riesgo sus poblaciones, desconociéndose los efectos que pueda sufrir el hábitat forestal por la repetida cosecha de estos productos (Alvarado y Benítez 2009).

Los artesanos de Tenancingo, México, conocen y utilizan diferentes especies vegetales para elaborar cestería (Saldívar et al. 2006), pero las piezas de mayor calidad provienen de los tallos de D. lutea. Debido a la demanda, el cambio de uso del suelo y la expansión de la floricultura, cada día es más difícil encontrar plantas de esta especie en los bosques de la localidad, lo que encarece la materia prima y disminuye el ingreso familiar. No existen a la fecha estudios que permitan determinar y evaluar la sostenibilidad de la recolecta de tallos de esta especie.

El objetivo del presente trabajo fue analizar la dinámica poblacional, el comportamiento anual y evaluar la sostenibilidad ecológica de una población de $D$. lutea (Cav.) Willd.

\section{MATERIALES Y MÉTODOS}

\section{Especie en estudio}

Dalea lutea (Cav.) Willd. (Fabaceae), localmente conocida como "romerillo" (Figura 1), es una planta arbustiva, de tallo difuso y pubescente; hojas compuestas; flores en espigas de color amarillo pálido; el fruto es una legumbre con una semilla reniforme, de 1-1,5 $\mathrm{mm}$ de ancho por 1,7-2,0 $\mathrm{mm}$ de largo de color amarillo obscuro y número de semillas por gramo de 1000 (López et al. 2005). Especie polimórfica de amplia distribución en regiones templadas de México, se encuentra asociada al bosque mixto, matorral y pastizal. Se distribuye desde Chihuahua, Coahuila y Nuevo León hasta Querétaro, Estado y Valle de México (Arreguín et al. 1997, Calderón y Rzendowski 2001). Martínez y Matuda (1979), la consideran de escaso valor, pero los tallos son utilizados para elaborar cestería en la región sur del Estado de México (Saldívar y Bernal 1998, Saldívar et al. 2006). 


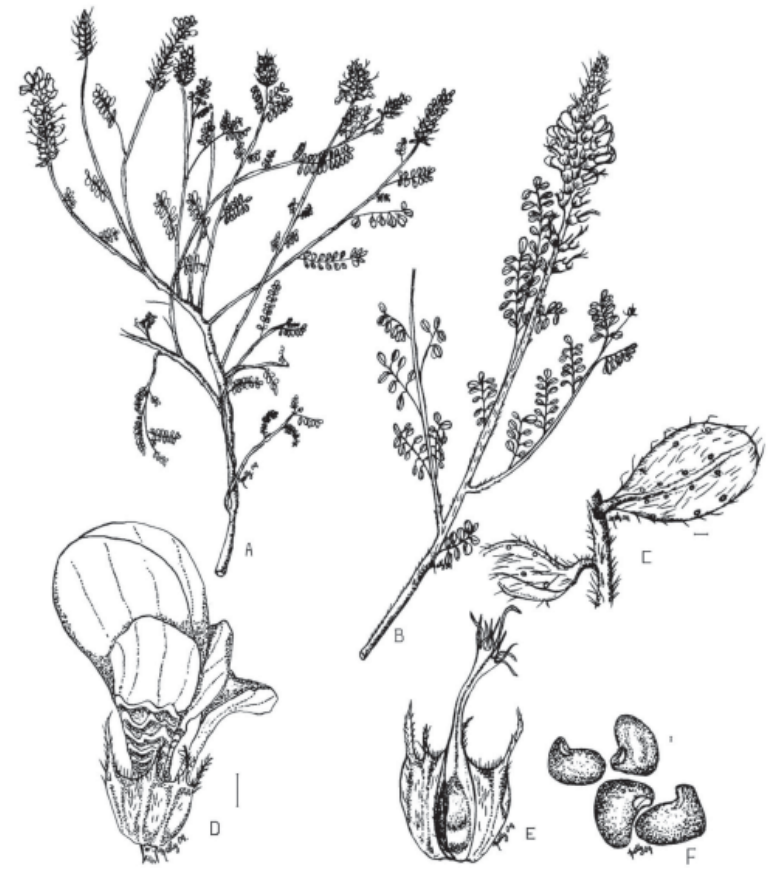

Figura 1. Dalea lutea (Cav.) Willd. A. Planta completa; B. Rama con hojas e inflorescencias; C. Hoja; D. Flor; E. Pistilo; F. Semillas. Tenancingo, Estado de México, México. 2002-2004.

\section{Área de estudio}

La investigación se llevó a cabo en el municipio de Tenancingo, localizado al sur este del Estado de México, en México (Figura 2), entre los $18^{\circ} 42^{\prime}$ y $19^{\circ} 05^{\prime}$ de latitud norte y $99^{\circ} 15^{\prime}$ y $99^{\circ} 50^{\prime}$ de longitud oeste y con una extensión de 160,2 km² (INEGI 2001b, López 2001), durante los años del 2002 al 2004. La ciudad de Tenancingo, capital del municipio del mismo nombre se encuentra situada a los $18^{\circ} 57^{\prime} 41,8^{\prime \prime}$ latitud norte y $99^{\circ} 35^{\prime} 36^{\prime \prime}$ longitud oeste a una altura de $2038 \mathrm{msnm}$. El clima predominante es templado subhúmedo con lluvias en verano; la temperatura media anual es de $17,3^{\circ} \mathrm{C}$, con mínima de $2^{\circ} \mathrm{C}$; la precipitación media es de 1262,6 mm anuales (García 1988, López 2001). En cuanto al uso del suelo, el 58\% es agrícola, el bosque representa el 34\%, la zona urbana ocupa el $7 \%$ y sólo una pequeña fracción es selva baja caducifolia. Los suelos predominantes son del tipo andosol, derivados de cenizas volcánicas, ácidos, ligeros y susceptibles a la erosión (INEGI 2001a). El paisaje está conformado

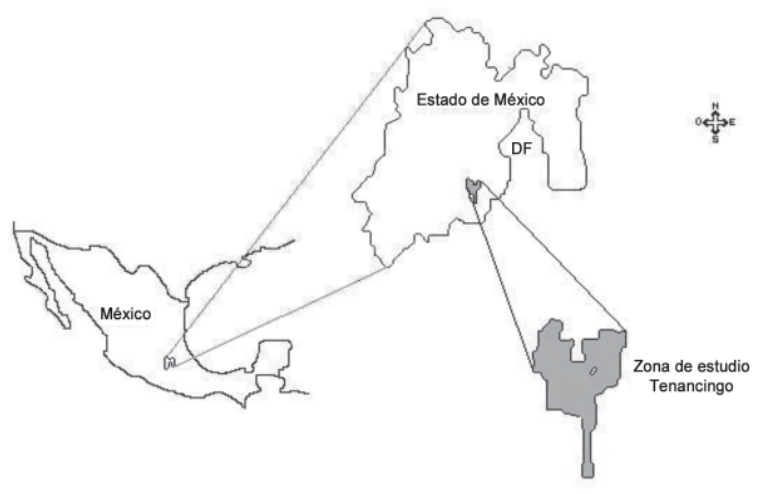

Figura 2. Zona de estudio: municipio de Tenancingo, Estado de México, México. 2002-2004.

por campos de cultivo, bosque mixto y pastizales, el bosque mix to está formado por especies de los géneros Pinus y Quercus, como Pinus leiophylla Schiede ex Schltdl. \& Cham., P. oocarpa Schiede ex Schltdl., P. michoacana Martínez, P. montezumae Lamb., P. teocote Schltdl. \& Cham., Quercus laurina Bonpl., Q. rugosa Née, Q. crassipes Humb. \& Bonpl. y Q. crasifolia Humb. \& Bonpl.; principalmente. La zona de pastizal se formó como consecuencia de la eliminación de la vegetación original y se caracteriza por la presencia de comunidades constituidas por especies de Bouteloua gracilis, Sporobolus sp., Digitaria sp., Muhlenbergia rigida, Aristida divaricata y otras que son manejadas para sostener una ganadería extensiva (Anónimo 2001, Rzedowski 1998). Según el XI Censo General de Población 2010, el municipio cuenta con 90946 habitantes (INEGI 2011), cuya actividad económica primaria se centra en la producción de flores, legumbres, cereales, frutas y forrajes; otras actividades importantes son la elaboración de artesanías, sobresaliendo los muebles de madera, textiles y cestería; en esta actividad artesanal sobresalen los poblados de San Martín, La Mesita y Chalchihuapán, siendo en esta comunidad en donde se encuentran el mayor número de cesteros; otras actividades importantes del municipio son el comercio y el turismo (Ayuntamiento de Tenancingo 2009).

\section{Ubicación de parcelas}

Dado que $D$. lutea tiende a crecer en manchones, se designaron de manera aleatoria ocho parcelas circulares de $200 \mathrm{~m}^{2}$ en terrenos ejidales cercanos a la comunidad 
de Chalchihuapán, municipio de Tenancingo (localizada a $18^{\circ} 57^{\prime} 37,5^{\prime \prime}$ latitud norte y $99^{\circ} 35^{\prime} 36,7^{\prime \prime}$ longitud oeste y a $2055 \mathrm{msnm}$ ); cuatro situadas en el bosque mixto y cuatro en la zona de pastizal inducido, la zona de estudio estuvo comprendida entre los $18^{\circ} 59^{\prime} 3,5^{\prime \prime}$ y $18^{\circ} 59^{\prime} 16,5^{\prime \prime}$ latitud norte y entre $99^{\circ} 33^{\prime} 41,1^{\prime \prime}$ y $99^{\circ} 33,2^{\prime \prime}$ longitud oeste (Cuadro 1). Las parcelas se subdividieron en cuatro cuadrantes de $50 \mathrm{~m}^{2}$, a modo de repeticiones. Las parcelas se mantuvieron expuestas al aprovechamiento de tallos y pastoreo, así como a los factores climáticos y al daño por incendios. El número de individuos de $D$. lutea se monitoreó durante nueve fechas, tomando como periodo de muestreo el inicio de las estaciones del año. La primera observación se efectuó durante el otoño del año 2002 y la última durante el otoño del 2004. En cada parcela se marcaron y registraron en un croquis de campo todos los individuos, agrupándolos en las siguientes clases:

Plántulas. Individuos germinados durante la primavera y verano, con una altura de $10-50 \mathrm{~cm}$, con un solo tallo.

Juveniles. Individuos con altura superior a $50 \mathrm{~cm}$. y menor a un metro, con tres a cuatro ramas laterales e indicios de haber sido aprovechadas el verano anterior.

Adultas. Individuos productivos, con apariencia de arbustos, altura superior a un metro, con ramificaciones terciarias y evidencia de haber sido cosechados en años anteriores.

\section{Longevidad de las semillas de $D$. lutea}

En el mes de febrero 2012 y para conocer la longevidad de las semillas, se montó un ensayo colocando
100 semillas de $D$. lute $a$ Willd., (repetidas cinco veces) en cajas Petri dentro de una germinadora a $22 \pm 1^{\circ} \mathrm{C}$ para determinar el porcentaje de germinación y la pérdida de viabilidad durante siete años.

Dinámica y comportamiento de la población de $D$. lutea, creciendo bajo condiciones de bosque mixto y pastizal.

Los resultados se muestran como total de individuos y por clase en una hectárea y fueron graficados y analizados de manera general (total y por clase) comparándolos con las precipitaciones medias de la zona (Anónimo 2005). Posteriormente, los datos se sometieron a un análisis de varianza de un arreglo bifactorial, donde los niveles de estudio fueron nueve épocas (muestreo al inicio de cada estación) y dos zonas de muestreo (pastizal y bosque mixto), con cuatro repeticiones; cuando hubo diferencias estadísticas, se aplicó la prueba de separación de medias de diferencia mínima significativa (DMS a $=0,05$ ). El análisis estadístico se hizo mediante el programa SAS (Rebolledo 2002, SAS 1985).

\section{Sustentabilidad de una población natural de $D$. lutea}

Se utilizó la metodología y las fórmulas descritas por Hall y Bawa (1993), quienes proponen el uso de tasas de restablecimiento y mortalidad (incremento o decremento) en poblaciones sometidas a recolecta, como índices positivo o negativo de la sostenibilidad.

Cuadro 1. Ubicación de las parcelas en estudio. Tenancingo, Estado de México, México. 2002.

\begin{tabular}{lcclc}
\hline Zona & Parcela & Latitud N & Longitud W & msnm \\
\hline Bosque mixto & 1 & $18^{\circ} 59^{\prime} 3,5^{\prime \prime}$ & $99^{\circ} 33^{\prime} 41,1^{\prime \prime}$ & 2220 \\
& 2 & $18^{\circ} 59^{\prime} 3,9^{\prime \prime}$ & $99^{\circ} 33^{\prime} 40,2^{\prime \prime}$ & 2227 \\
& 3 & $18^{\circ} 59^{\prime} 6,0^{\prime \prime}$ & $99^{\circ} 33^{\prime} 34,9^{\prime \prime}$ & 2229 \\
& 4 & $18^{\circ} 59^{\prime} 7,1^{\prime \prime}$ & $99^{\circ} 33^{\prime} 34,1^{\prime \prime}$ & 2234 \\
\hline Pastizal & 1 & $18^{\circ} 59^{\prime} 12,7^{\prime \prime}$ & $99^{\circ} 33^{\prime \prime} 33,3^{\prime \prime}$ & 2251 \\
& 2 & $18^{\circ} 59^{\prime} 13,9^{\prime \prime}$ & $99^{\circ} 33^{\prime} 34,0^{\prime \prime}$ & 2264 \\
& 3 & $18^{\circ} 59^{\prime} 16,5^{\prime \prime}$ & $99^{\circ} 33^{\prime} 36,8^{\prime \prime}$ & 2265 \\
& 4 & $18^{\circ} 59^{\prime} 16,5^{\prime \prime}$ & $99^{\circ} 33^{\prime} 33,2^{\prime \prime}$ & 2277 \\
\hline
\end{tabular}


Para el restablecimiento (ri) se usó la fórmula:

$$
r i=100\left\{\frac{\ln [n o+a i / n o]}{t i}\right\}
$$

Donde:

$\ln =$ logaritmo .

no $=$ número de individuos al primer recuento .

$a i=$ número de individuos adicionales a la población al segundo recuento.

$t i=$ número de años entre conteos.

La mortalidad ( $\boldsymbol{m i}$ ) se calculó con la fórmula:

$$
m i=100\left\{\frac{\ln [n o+a i / n o]}{t i}\right\}
$$

Donde:

$\ln =\log$ aritmo

no $=$ número de individuos al primer conteo

$a i=$ número de individuos muertos durante intervalo de estudio

$t i=$ número de años entre conteos.

Para evaluar la sostenibilidad poblacional, tanto cuando la especie estuvo asociada al bosque mixto como cuando se desarrolló en pastizal inducido, se utilizaron los promedios del total de individuos presentes en una hectárea durante la primera, intermedia y última observación. Se efectuó un estudio por clases y se determinó la sostenibilidad. Los datos se presentan como índices porcentuales de sostenibilidad o no sostenibilidad.

\section{RESULTADOS Y DISCUSIÓN}

\section{Longevidad de las semillas de $D$. lutea}

En un ensayo llevado a cabo por López et al. (2005) encontraron porcentajes germinación para las semillas de esta especie de 37,3 cuando no aplicaron ningún tratamiento pre germinativo. En el presente ensayo y con semilla del mismo lote, pero con una antigüedad de siete años, se encontraron porcentajes promedio de 17,4 con una desviación estándar de \pm $2,3 \%$, esto muestra que la semilla pierde un $2,8 \%$ de su capacidad germinativa por año.

\section{Dinámica y comportamiento poblacional de $D$. lutea por zona}

El comportamiento de la clase plántula de $D$. lutea tanto en el bosque mixto como en el pastizal fue similar (Figuras 3 y 4), pero en el primero se observó un ligero aumento para el muestreo de invierno 2002, mientras que en el pastizal inducido disminuía; fenómeno

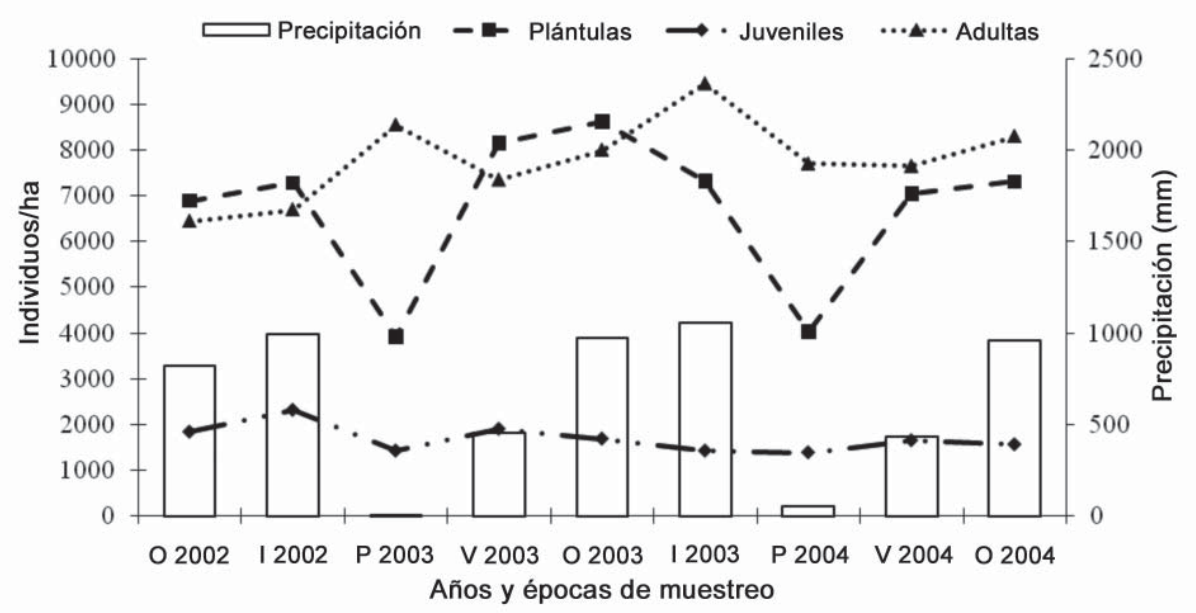

Figura 3. Dinámica y comportamiento de D. lutea (Cav.) Willd., creciendo bajo condiciones de bosque mixto. Tenancingo, Estado de México. México. 2002-2004. 


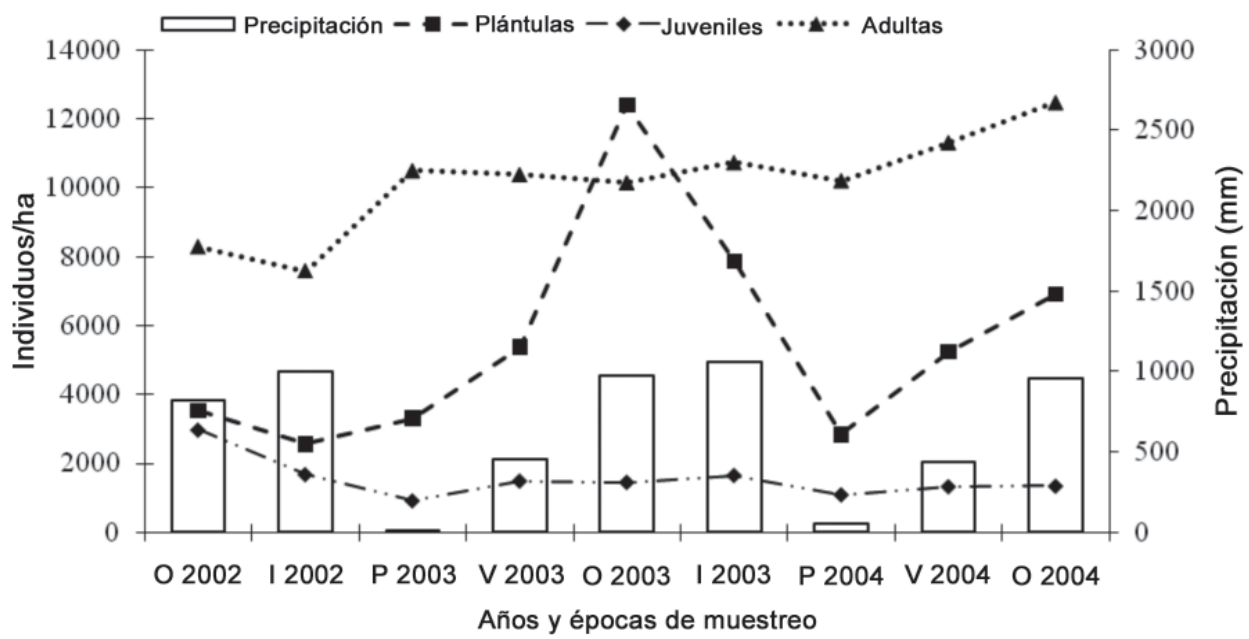

Figura 4. Dinámica y comportamiento de D. lutea (Cav.) Willd., creciendo bajo condiciones de pastizal inducido al eliminar la vegetación original. Tenancingo, Estado de México. México. 20022004.

contrario sucedió durante el muestreo de primavera 2003 donde se observó un repunte en el número de plántula dentro del pastizal inducido.Para los muestreos de verano y otoño 2003 esta clase mostró la misma tendencia en ambas zonas de estudio, pero el número de individuos en el pastizal inducido fue muy alto; durante ese invierno, ambas zonas presentaron disminución de plántulas. Una tendencia similar se presentó durante los tres últimos muestreos efectuados durante el año 2004, donde esta clase presenta un mínimo en primavera con aumento gradual para el verano y con un máximo en otoño. Es de resaltar que comparando el número de plántulas durante el muestreo de otoño 2003, el pastizal inducido superó ampliamente al bosque mixto.

Durante la investigación se observó que las plántulas determinan el comportamiento general de la población y conforme se sucedieron los muestreos, la población disminuyó durante el invierno hasta un mínimo en primavera (Figura 3), repuntando durante el verano y otoño. Esto se debe a factores limitantes durante el invierno y la primavera, que en la zona se caracterizan por bajas temperaturas y estiaje (octubremayo); pero cuando aumenta la temperatura y se establece el régimen de lluvias (junio-septiembre), se favorecen la germinación y el crecimiento de plantas.

De las clases en estudio, las plántulas son las más susceptibles, pero al mismo tiempo constituyen la clase con mayor dinamismo y la que se recupera más fácilmente, esto se puede deber a la gran cantidad de semilla que producen las plantas adultas y que permanece latente para las próximas temporadas de germinación, debido a que las semillas conservan su viabilidad y el "banco" de semillas en el suelo aumenta con los años.

El comportamiento de las juveniles en bosque mixto (Figura 3) sólo presentó elevación durante el muestreo de invierno 2002, con un ligero decremento para la primavera 2003 y valores estables durante los seis últimos muestreos. Por su parte, esta misma clase en la zona de pastizal inducido (Figura 4), presentó valores altos durante el primer muestreo, mismos que decrecieron para el de invierno 2002 llegando a un mínimo durante la primavera 2003, y manteniendo valores estables para el resto de los muestreos.

Aunque los juveniles mostraron una dinámica similar, se vieron afectados de forma más severa y en mayor porcentaje que las plántulas. La disminución pudo deberse al paso a la clase adulta o a factores externos. Para que las juveniles logren pasar a la clase adulta se requieren de dos a tres años, tiempo en el cual están expuestas a los mismos factores adversos que las plántulas con los agravantes de que en condiciones de bosque mixto el crecimiento se hace lento por sombreado, mientras que en pastizal las plantas son consumidas por ovinos.

La clase adulta en bosque mixto (Figura 3) presentó un comportamiento ascendente durante los muestreos de otoño 2002 y primavera 2003 con ligero decremento 
para el verano 2003 y una tendencia ascendente para los dos últimos muestreos del 2003, durante el año 2004 presentó estabilidad numérica. Por su parte, esta misma clase en pastizal inducido (Figura 4), tuvo un comportamiento ascendente, ya que el promedio menor se reportó durante el primer muestreo, y el mayor con el último (otoño 2002 y 2004, respectivamente). Cuando la planta alcanza el estado adulto, aumenta su capacidad de supervivencia y es capaz de tolerar daño por frío, incendio, sobrepastoreo y colecta de tallos. Además, la población se mantiene presente, pese a la extracción de plantas que hacen los habitantes del lugar. La permanencia y presencia de la clase adulta, explica la perennidad y resistencia de la especie.

En lo que respecta al comportamiento de la población de $D$. lutea dentro de las zonas de estudio y aún cuando las medias no presentan diferencias estadísticas, se pudo notar ligeramente un número mayor de individuos de las tres clases cuando la especie creció asociada con el bosque de pino-encino, y aunque la especie se desarrolla bien en ambos ambientes, parece estabilizarse mejor en este último espacio (Cuadro 2).

\section{Evaluación poblacional global}

En el caso de plántulas, el análisis de varianza mostró diferencias significativas $(\mathrm{P}>0,01)$ para fechas de muestreo, no así para zonas o en la interacción fechas por zonas. Algo similar se encontró en las juveniles donde solo hubo diferencia $(\mathrm{P}>0,05)$ en fechas de muestreo. En la clase adulta no se detectaron diferencias para alguno de los factores de estudio.

La media más alta para plántulas de $D$. lutea correspondió al otoño 2003 (Cuadro 3), superior y

Cuadro 2. Promedio de plántulas, juveniles y adultas de $D$. lutea bajo condiciones de bosque mixto y de pastizal inducido al eliminar la vegetación original. Tenancingo, Estado de México. México. 2002-2004.

\begin{tabular}{lccc}
\hline Localidad de muestreo & Plántulas (ha) & Juveniles (ha) & Adultas (ha) \\
\hline Bosque mixto & 6602 a & 1727 a & 2177 a \\
Pastizal & 5559 a & 1555 a & 1948 a \\
Diferencia mínima & 1788 & 351 & 462 \\
\hline
\end{tabular}

Datos con la misma literal en la columna, no difieren significativamente (DMS a $=0,05$ ).

Cuadro 3. Promedio de plántulas, juveniles y adultas de $D$. lutea en bosque mixto y en pastizal inducido al eliminar la población original. Tenancingo, Estado de México. México. 2002-2004.

\begin{tabular}{lccc}
\hline Muestreos & Plántulas (ha) & Juveniles (ha) & Adultas (ha) \\
\hline Otoño 2002 & $5106 \mathrm{bc}$ & $2406 \mathrm{a}$ & $1693 \mathrm{a}$ \\
Invierno 2002 & $4925 \mathrm{bc}$ & $2000 \mathrm{ab}$ & $1650 \mathrm{a}$ \\
Primavera 2003 & $3619 \mathrm{c}$ & $1175 \mathrm{c}$ & $2193 \mathrm{a}$ \\
Verano 2003 & $6763 \mathrm{abc}$ & $1700 \mathrm{abc}$ & $2031 \mathrm{a}$ \\
Otoño 2003 & $10513 \mathrm{a}$ & $1587 \mathrm{bc}$ & $2087 \mathrm{a}$ \\
Invierno 2003 & $7594 \mathrm{ab}$ & $1543 \mathrm{bc}$ & $2331 \mathrm{a}$ \\
Primavera 2004 & $3438 \mathrm{c}$ & $1412 \mathrm{bc}$ & $2056 \mathrm{a}$ \\
Verano 2004 & $6113 \mathrm{bc}$ & $1493 \mathrm{bc}$ & $2150 \mathrm{a}$ \\
Otoño 2004 & $6661 \mathrm{bc}$ & $1456 \mathrm{bc}$ & $2375 \mathrm{a}$ \\
Diferencia mínima & 3794 & 744 & 980 \\
Media general & 6081 & 1641 & 2063 \\
\hline
\end{tabular}

Datos con la misma literal en la columna, no difieren significativamente (DMS a $=0,05$ ). 
estadísticamente diferente a lo encontrado para los otoños 2002 y 2004 , pero similar a los valores encontrados del verano e invierno del 2003; los valores de invierno 2002 y verano 2004 fueron menores, pero no difirieron entre sí; los promedios más bajos fueron los reportados durante las primaveras 2003 y 2004. El valor más alto se alcanzó durante el otoño 2003 y el más bajo en la primavera 2004. En la clase juvenil el promedio más alto fue en otoño 2002, superior y estadísticamente diferente a los de otoño 2003 y 2004. Los promedios de los muestreos de invierno 2002 y primavera 2003 no difieren estadísticamente de lo reportado para el primer muestreo. A partir del otoño 2003 y hasta la última observación se formó un grupo de medias que no difirieron estadísticamente entre sí. El valor más bajo fue durante la primavera 2003; de manera general se pudo observar una tendencia a la baja durante el tiempo de observaciones. En la clase adulta, aún cuando no hubo diferencias significativas, se notó un aumento gradual en sus individuos a través de temporadas.

Según las medias generales se puede notar que la población presenta una composición de 21, 17 y $62 \%$ de individuos de las clases adultas, juveniles y plántulas, respectivamente.

\section{Sostenibilidad de $D$. lutea en bosque mixto y pasti- zal inducido}

De manera general, se encontraron diferencias en establecimiento o mortalidad dependiendo de la clase en estudio.

Plántulas. Durante el primer año de observación (Cuadro 4), se notaron porcentajes de restablecimiento de esta clase tanto en bosque mixto como en pastizal inducido, pero el restablecimiento fue menor en la primera zona que en la segunda. Durante el segundo año (Otoño 2003-2004) la clase plántulas presentó mortalidad en ambas zonas de estudio, siendo mayor en la zona de pastizal inducido, donde las pérdidas alcanzaron porcentajes más altos. Tomando en consideración el periodo de evaluación, esta clase presentó un decremento de $4,4 \%$ en el bosque mixto y un restablecimiento de $59,5 \%$ en el pastizal inducido. Los resultados diferenciales se pueden deber a las condiciones de iluminación, efecto de incendio y al banco de semillas existente en el suelo que prevalecen en una y otra condición de estudio.

Juveniles. Durante el primer año de observación (Otoño 2002-2003), esta clase presentó valores negativos en ambas zonas de estudio, solo que cuando se evaluó el número de individuos en el pastizal inducido, este presentó un valor negativo muy alto. Durante el segundo periodo (Otoño 2003-2004) se notaron porcentajes de pérdida similares en ambas zonas de estudio, pero menores que el año anterior. La evaluación global de la clase juveniles mostró que, la clase disminuyó en 16,2\% anual en la zona de bosque mixto y $79 \%$ en la de pastizal inducido (Cuadro 4). El decremento en juveniles muestra que esta clase es la más vulnerable a condiciones adversas del crecimiento y requiere de más de dos años para pasar de ese estado al de planta adulta.

Adultas. En el periodo comprendido entre el otoño 2002 al 2003, se puede notar (Cuadro 4) que en ambas zonas se restableció la clase adulta, con valores porcentuales similares. No obstante, en el siguiente año (Otoño 2003-2004) el restablecimiento se mantuvo con valores positivos, pero presentó una fuerte caída en bosque mixto, mientras que en la zona de pastizal inducido se mantuvo casi igual que en el año anterior. Analizando el comportamiento de la clase adulta en las

Cuadro 4. Índices de restablecimiento/mortalidad (\%) de una población de D, lutea (Cav,) Willd, creciendo asociada a un bosque mixto y en pastizal inducido al eliminar la población original. Tenancingo, Estado de México, México, 2002-2004.

\begin{tabular}{lcccccc}
\hline \multirow{2}{*}{$\begin{array}{l}\text { Periodo de } \\
\text { evaluación }\end{array}$} & \multicolumn{2}{c}{ Clase plántulas } & \multicolumn{2}{c}{ Clase juveniles } & \multicolumn{2}{c}{ Clase adultas } \\
\cline { 2 - 7 } & $\begin{array}{c}\text { Bosque } \\
\text { Mixto }\end{array}$ & $\begin{array}{c}\text { Pastizal } \\
\text { Inducido }\end{array}$ & $\begin{array}{c}\text { Bosque } \\
\text { Mixto }\end{array}$ & $\begin{array}{c}\text { Pastizal } \\
\text { Inducido }\end{array}$ & $\begin{array}{c}\text { Bosque } \\
\text { Mixto }\end{array}$ & $\begin{array}{c}\text { Pastizal } \\
\text { Inducido }\end{array}$ \\
\hline Otoño 02-03 & $+25,4$ & $+125,7$ & $-7,04$ & $-71,0$ & $+21,5$ & $+20,3$ \\
Otoño 03-04 & $-29,8$ & $-66,2$ & $-9,16$ & $-8,0$ & $+3,6$ & $+20,7$ \\
Diferencia & $-4,4$ & $+59,5$ & $-16,2$ & $-79,0$ & $+25,1$ & $+41,0$ \\
\hline
\end{tabular}


dos zonas de estudio durante el tiempo de evaluación, se puede notar que en el pastizal inducido el porcentaje de recuperación es superior $(41 \%)$ al calculado para bosque mixto $(25,1 \%)$. El aumento de individuos de esta clase permite afirmar que la población es exitosamente sostenible en la zona de pastizal inducido y se mantiene presente en la zona de bosque mixto. La estabilidad e incremento de la clase adulta se debe a la incorporación de juveniles, así como, a la resistencia de las adultas a condiciones adversas.

Al analizar y comparar los índices de sostenibilidad de la especie cuando creció asociada a bosque mixto y pastizal inducido, se notó que dentro del primer tipo de vegetación el restablecimiento, cuando lo hubo, fue menor al encontrado para pastizal inducido, aun y cuando la pérdida de juveniles en esta zona fue menor. Por otro lado, aunque la pérdida de juveniles fue muy alta en pastizal inducido, el aumento de adultas fue sobresaliente. Esto puede deberse a que las tres clases de Dalea lutea en bosque mixto se relacionan y compiten con otras especies por espacio, agua y nutrientes; mientras que en el pastizal solo se encuentran asociadas con gramíneas; aunado a lo anterior, durante el invierno de 2002, en tres de las cuatro parcelas del pastizal se presentaron incendios, lo que eliminó tanto a gramíneas como a plántulas y juveniles.

Endres et al. (2006) encontraron índices de restablecimiento poblacional de 1,11 para Chamaedorea radicalis después de seis años de cuantificar la recolecta de hojas; por otro lado, diferentes porcentajes de sobrevivencia y crecimiento desde 0,005 hasta 1,11 en Phytelephas aequatorialis sometidas a diferentes formas de manejo y solo encontró sostenibilidad cuando la palma creció en parcelas secas y limpias (Velásquez 1998). Los resultados antes encontrados, difieren de los de este trabajo en cuanto a la especie estudiada como en la parte de la planta utilizada, ya que en el presente caso son tallos la parte de interés y el estudio se llevó a cabo en condiciones naturales. Los resultados de esta investigación son similares a los encontrados por Marshall y Newton (2003) en el caso de la recolecta de frutos de Tila (Ternstroemia lineata) donde los árboles adultos de esta especie no difirieron en número en zonas cercanas o lejanas de los núcleos de población de los recolectores, indicando que la cosecha, desde una perspectiva ecológica, no puede considerarse como no sustentable.
La sostenibilidad de D. lutea se ve favorecida porque la parte utilizada de la planta son tallos en pleno crecimiento, previo a la floración, fructificación, maduración y dispersión de semillas; con lo cual no se ven afectados los procesos de propagación natural; caso contrario en magueyes para la producción de mezcal, donde se utiliza la planta completa y las poblaciones silvestres se ven disminuidas e incluso comienzan a presentar extinciones locales debido a la demanda del producto (Barraza y Estrella 2008). Londoño (2006) menciona que la sobreexplotación de un recurso imposibilita su uso para futuras generaciones o impide su implementación en otros aprovechamientos, no siendo el caso que nos ocupó.

Las pérdidas o restablecimientos de la clase plántulas determinan la respuesta poblacional, - positiva o negativa -, a las condiciones de precipitación, siendo más favorables durante el verano y parte del otoño, cuando se presentan la germinación y el crecimiento vegetativo. Por otro lado, el decremento en juveniles muestra que esta clase es la más vulnerable a condiciones adversas del crecimiento. La estabilidad e incremento de la clase adulta se debe a la incorporación de juveniles, así como, a la resistencia de las adultas a condiciones adversas.

La mejor época para el aumento poblacional coincide con verano y otoño. De acuerdo a la información y bajo las condiciones actuales, la recolecta de tallos de $D$. lutea para elaborar cestería se considera sustentable y los índices de restablecimiento para las clases plántulas y adultas fueron mayores cuando la población estuvo asociada al pastizal inducido. Aún así, se requiere evaluar diferentes intensidades de recolecta, lo cual puede hacerse en parcelas experimentales a fin de generar condiciones controladas. Los resultados permiten deducir que la presión de recolecta, los incendios, el aprovechamiento del bosque y la disminución de terreno forestal no parecen afectar significativamente a la población vegetal y ésta se mantiene presente en el bosque.

\section{AGRADECIMIENTOS}

Los autores agradecen a la Universidad Autónoma del Estado de México y a la Secretaría de Investigación 
y Estudios Avanzados por el apoyo otorgado para la elaboración de la presente investigación.

\section{LITERATURA CITADA}

Alvarado, CG; Benítez G. 2009. El enfoque de agroecosistemas como una forma de intervención científica en la recolección de hongos silvestres comestibles. Tropical and Subtropical Agroecosystems 10(3):531-539.

Anónimo. 2001. Especies con usos no maderables en bosques tropicales y subtropicales en los Estados de Durango, Chihuahua, Jalisco, Michoacán, Guerrero y Oaxaca (en línea). SEMARNAT-PROCYMAF. Consultado 30 de julio 2008. Disponible en http://www. semarnat.gob.mx/pfnm2/.html

Anónimo. 2005. Precipitaciones 2002, 2003, 2004, Estación San Bartolito Tenancingo. Comisión Nacional del Agua, Residencia Estado de México. Archivos electrónicos. Toluca, Estado de México, México.

Arreguín, SMG; Cabrera LR; Fernández NC; Orozco LB; Rodríguez CM; Yépez B. 1997. Introducción a la flora del estado de Querétaro. CCTEQ, IPN y UACh. Santiago de Querétaro, México. 361 p.

Ayuntamiento de Tenancingo. 2009. Plan municipal de desarrollo urbano 2009-2012. Actividades económicas locales. Tenancingo, Estado de México. p. 26-33.

Barraza, RE; Estrella, RJP. 2008. Manejo sustentable de los recursos naturales guiado por proyectos científicos en la mixteca poblana mexicana. Ecosistemas 17(2):3-9.

Calderón, RG; Rzedowski, J. (eds.). 2001. Flora fanerogámica del Valle de México. 2 ed. Instituto de Ecología, A. C. y Comisión Nacional para el Conocimiento y Uso de la Biodiversidad. Pátzcuaro, Michoacán. México. 1406 p.

Carabias, LJE; Provencio, D; Toledo, MC. 1995. Manejo de recursos naturales y pobreza rural. Universidad Nacional Autónoma de México - Fondo de Cultura Económica. México, D. F. 138 p.

Casas, MA. 1996. Reflexiones sobre el desarrollo agrícola sostenible. In J. Pérez; R. Cerrato. eds. Nuevos horizontes en agricultura, agroecología y desarrollo sostenible. Colegio de Postgraduados. Montecillo, Estado de México. 256-262 p.

Endres AB; Gorchov D; Barry EJ. 2006. Sustainability of non-timber forest product: Effects of alternative leaf harvest practice over 6 years on yield and demography of the palm Chamaedorea radicalis. Forest Ecology and Management 234:181-191.
García, E. 1988. Modificaciones al sistema de clasificación climática de Köppen. Instituto de Geografía. Universidad Nacional Autónoma de México. México, D. F. $246 \mathrm{p}$.

Gómez, PM; Angón, TM 2004. Recursos forestales no maderables aprovechados en Morelia. Serie Fuentes para el conocimiento natural de Michoacán. Gobierno del Estado de Michoacán. Morelia, Michoacán, México. p. 17-23.

Hall, P; Bawa, K. 1993. Methods to assess the impact of extraction of non-timber tropical forest products on plant populations. Economic Botany 47:234-247.

INEGI (Instituto Nacional de Estadística, Geografía e Informática). 2001a. Síntesis de información geográfica del estado de México. Instituto Nacional de Estadística, Geografía e Informática. Aguascalientes, Ags. México. $139 \mathrm{p}$.

INEGI (Instituto Nacional de Estadística, Geografía e Informática). 2001b. Síntesis de información geográfica del estado de México. Instituto Nacional de Estadística, Geografía e Informática. Aguascalientes, Ags. México. Anexo Cartográfico. Carta Topográfica.

INEGI (Instituto Nacional de Estadística, Geografía e Informática). 2011. Censo de Población y Vivienda 2010. Instituto Nacional de Estadística, Geografía e Informática (en línea). Consultado 1 julio 2011. Disponible en http: www.inegi.org.mx

Kammerbauer, J. 2001. Las dimensiones de la sostenibilidad: Fundamentos ecológicos, modelos paradigmáticos y senderos. Interciencia 26(8):353-359.

Londoño, CL. 2006. Los recursos naturales y el medio ambiente en la economía de mercado. Revista Científica Guillermo de Ockham 4(1):25-42.

López, MJ. 2001. Tenancingo monografía municipal. Instituto Mexiquense de Cultura. Gobierno del Estado de México. Toluca. Estado de México. 124 p.

López, GF, Enríquez LC; Saldívar, IP. 2005. Evaluación de la estratificación de frio-húmedo y escarificación mecánica en "Semillas" de Dalea lutea (Cav.) Willd. Revista Ciencias Agrícolas Informa (15):58-62.

Marshall, E; Newton, AC. 2003. Non timber forest products in the community of El Terrero, Sierra de Manantlán Biosphere Reserve, México: Is their Use Sustainable? Economic Botany 57(2): 262-278.

Martinez, M; Matuda, E. 1979. Flora del estado de México. Las leguminosas. Tomo II. Edición Facsimilar de los fascículos publicados en los años 1953 a 1972. Biblioteca Enciclopédica del Estado de México. Editorial Libros de México, S. A. México, D. F. p. 1-62. 
Matteucci, S; Colma, A. 1997. Agricultura sostenible y ecosistemas áridos y semiáridos de Venezuela. Interciencia 22(3):123-130.

Pimentel, PCO; Castañeta, VV. 2007. Estado del la conservación de las especies vegetales utilizadas para la artesanía en el valle de San Andrés, Pinar del Río, Cuba. Quebracho. Revista de Ciencias Forestales 14:90-98.

Rebolledo RHH. 2002. Manual SAS por computadora. Análisis estadístico de datos experimentales. Editorial Trillas, México, D. F. 52-100 p.

Rzedowski, J. 1998. Vegetación de México. Séptima reimpresión. Editorial Limusa-Grupo Noriega Editores. México, D. F. 432 p.

Saldívar, IP; Bernal, FE. 1998. Diagnóstico de la utilización de la vara de romerillo (Dalea spp) en la comunidad de Chalchihuapán, Municipio de Tenancingo. Memorias del Seminario Mesoamericano Sobre Agrodiversidad en la Agricultura Campesina (28-30 de abril) Campus Universitario El Cerrillo, Facultad de Ciencias Agrícolas, UAEM. p. 172-178.
Saldívar, IP; Laguna, CA; Esquivel, AC; González, EC. 2006. Plantas silvestres con uso artesanal en el Centro de México. Mesoamericana 10(4):8-14.

SARH (Secretaría de Agricultura y Recursos Hidráulicos). 1994. Compendio estadístico de la producción forestal 1989-1993. Secretaria de Agricultura y Recursos Hidráulicos (en línea). Consultado 30 julio 2008. Disponible en http://www.siap.sagarpa.gob.mx/Biblioteca/ ar_regpubsiap.pdf

SAS (Statistical Analysis System). 1985. SAS/STAT Guide for personal computers. Version 6 Edition. SAS Institute Inc. Cary N. C. USA. 378 p.

Sundriyal, M; Sundriyal. R. 2001. Wild edible plants of the sikkim Himalaya: Nutritive values of selected species. Economic Botany 53(3):377-390.

Velázquez, RJ. 1998. Productivity and sustainnability of a vegetable ivory palm (Phytelephas aequatorialis, Arecaceae) under three management regimes in Northwestern Ecuador. Economic Botany 52(2):168182 . 